\title{
The Numerical Simulation of the Land-slide Surge Basing on SPH
}

\author{
Weihua $\mathrm{Hu}^{1, \mathrm{a}}$ \\ ${ }^{1}$ College of Science, Wuhan University of Science and Technology, Wuhan, China , 430081 \\ awhhuzcs@163.com
}

Keywords: Land-slide Surge ; SPH ; Submarine Landslide ; Aerial Landslide

Abstract. The Landslide Surge is a common natural disaster, which has the strong non-linear feature. In this paper, the SPH method is used to numerically simulate the generation and propagation of the surge which is caused by the sliding of Submarine and Aerial sliders in 2D. The comparison with the experimental data indicates that the SPH method is valid to simulate the generation and the propagation of the Landslide-generated surge. Also, numerical diffusion has been preliminarily discussed during the simulation.

\section{Introduction}

Landslides due to storms, earthquakes and erosion are common natural phenomena. Large-scale Landslides near water bodies such as river-ways and reservoir may generate huge surges which not only do instant harm to everything in the affected water areas, but with the propagation and the superposition of surges, cause some reservoir crashing as dam-breakings and debacles, and some sea-damages like channel-bumps and ship-capsizing.

The Landslide-generated surge has being remained a problem appealing to hydraulic professionals and engineering fields, on which numerous researchers have performed a lot of experimental studies and numerical simulations[1,2]. The numerical researches on early Landslide-generated surges are mainly based on the shallow water approximation[3,4]. From 1980s, some numerical studies on the base of the finite volume method were carried out extensively[5,6]. Because of the strong non-linear character of the Landslide-generated surge, the particle method on Lagrange method is extremely suitable for applied solutions. In recent years, Smooth Particle Hydrodynamic-SPH method was adopted to solve Landslide-generated surges problems in some literatures[7,8]. The feasibility of the solving by SPH has been elementarily discussed.

Some physical tests in literatures of Heinrich are taken as objects researched in this paper. The author adopted the weak compressive SPH method based on the turbulent model to numerically simulate the process in which Submarine and Aerial sliders slide along a slope. The generation and propagation of 2-D surge waves were simulated, analyzed and discussed. The numerical results were compared with the experimental results. At last, the numerical diffusion of the numerical simulation was preliminarily discussed.

\section{Numerical Method}

The SPH method describes the fluid system by a series of spatially distributed Lagrange particles, among which connection by meshes is not needed, to which some necessary physical information as density, pressure, internal energy and speed etc. has been given. In view of the turbulent characteristics existing in the Landslide-generated surge, the boundary layer should be taken into account and the sub-grid-turbulence model should be dealt. Approximate expressions for smooth particles in the form of the continuous equation and the momentum equation are as follows:

$$
\frac{d \rho_{i}}{d t}=\sum_{j=1}^{N} m_{j} v_{i j}{ }^{\beta} \cdot \frac{\partial W_{i j}}{\partial x_{i}{ }^{\beta}}
$$




$$
\frac{d v_{i}^{\alpha}}{d t}=-\sum_{j=1}^{N} m_{j}\left(\frac{P_{i}}{\rho_{i}^{2}}+\frac{P_{j}}{\rho_{j}^{2}}\right) \frac{\partial W_{i j}}{\partial x_{i}^{\alpha}}+\sum_{j=1}^{N} m_{j}\left(\frac{4_{0} \mathbf{x}_{i j} \mathbf{v}_{i j}}{\left(\rho_{i}+\rho_{j}\right) \mathbf{x}_{i j}^{2}}\right) \frac{\partial W_{i j}}{\partial x_{i}^{\beta}}+\sum_{j=1}^{N} m_{j}\left(\frac{\tau_{i}}{\rho_{i}^{2}}+\frac{\tau_{j}}{\rho_{j}^{2}}\right) \frac{\partial W_{i j}}{\partial x_{i}^{\alpha}}+g^{\alpha}
$$

$\alpha$ and $\beta$ denote the direction of coordinates and the superposition of equations, respectively; $\rho_{i}$ 、 $m_{i} 、 x_{i} 、 v_{i}$ and $P_{i}$ are density, mass, displacement, velocity and pressure of particle $i(i=1,2 \ldots$ $, N) ; N$ is the total number of particles in the compact support domain of the particle $i ; v_{i j}=v_{i}-v_{j}$ is the relative speed between the particle $i$ and the particle $j ; x_{i j}=x_{i}-x_{j} ; V_{0}$ is the motion viscosity coefficient of laminar flow; $\tau_{i}$ is the sub-particle shear force. $W_{i j}=W\left(x_{i}-x_{j}, h\right)$ is a kernel approximate function. In this paper, cubic spline kernel function was chosen as follows:

$$
W(R, h)=\alpha_{d} \times\left(\frac{2}{3}-R^{2}+\frac{1}{2} R^{3}\right), 0 \leq R<1 ; \alpha_{d} \times \frac{1}{6}(2-R)^{3} 1 \leq R<2 ; 02 \leq R
$$

where coefficient $\alpha_{d}$ is $\frac{15}{7 \pi h^{2}}$ in $2 \mathrm{D}, h$ is the smoothing length, representing the valid influence domain of the smoothing function. Usually, $h$ is set the product of the interval between particles multiplied by the smoothing length coefficient $h_{c}$, that is $h=h_{c} \sqrt{\Delta x^{2}+\Delta z^{2}}$.

\section{Landslide surge problem}

In this paper, a 2-D Landslide-generated surge is modelled with Heinrich[1] physical experiments regarded as computed projects. In the physical experiment, the slider is simplified to a right triangular solid slider, with the slope matching with the side wall. The experiment contains two states. The first is a Submarine Landslide in which the slider is initially submerged. The second is an Aerial Landslide in which the slider is initially above the still free-surface. This experimental model is shown in Fig. 1.

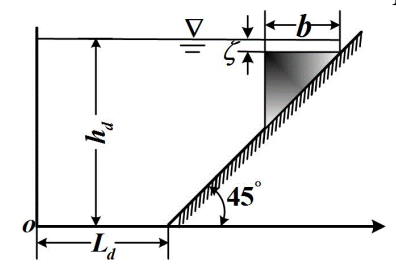

Fig.1 2D Landslide surge model

The reservoir has a 45 degree slope on which there is a wedge-shaped slider with the size $0.5 \mathrm{~m} \times$ $0.5 \mathrm{~m}$. The horizontal bottom of the reservoir is $18 \mathrm{~m}$ in length. For the Submarine Landslide, the water depth is $h_{d}=1 \mathrm{~m}$ and the top of the slider is initially located $\zeta=0.01 \mathrm{~m}$ below the free-surface. For the Aerial Landslide, the water depth $h_{d}=0.4 m$ and the bottom of the slider is just above the undisturbed free-surface at the beginning.

In the physical experiment, an obstacle is settled on the slope close to the bottom of the water. During the test, the slider freely slides down to the bottom and stops as it reaches the obstacle. In the numerical model, let the velocity of the slider zero after it reaches the coordinate point of the obstacle.

\section{Results}

Simulation of the Submarine Landslide. In the numerical model of the SPH method, the interval between particles $\Delta x=\Delta z=0.02 \mathrm{~m}$, the time step is $\Delta t=1 \times 10^{-5} \mathrm{~s}$, the density of water is $1000 \mathrm{~kg} / \mathrm{m}^{3}$, motion viscosity $v_{0}=1 \times 10^{-6} \mathrm{~m}^{2} / \mathrm{s}$.

The calculated model is set as follows: smoothing length coefficient $h_{c}=0.92$ is given. Boundary particles experience the Lennard-Jones repulsive force; particle pressure is realized by the weakly 
compressive state equation; the time integral is carried out by prediction correction method; searching for adjacent particles is achieved by linked lists.

Generation of the surge wave. In Fig.2, wave profiles obtained by the numerical simulation are within the region of the generation of the surge $16.0<x<20.0 m$ at a series time.

As seen in Fig. 2, the simulated wave profiles agree with experimental results within the region of the generation of the surge. During the initial phase of the sliding of the slider, since the quantity of particles, which is a significant factor of the error for the simulation of the generation of the surge, is rare on the top of the slider, it goes against the precision of the integral approximation of the adjacent fluid field of the slider. Hence increasing the amount of particles around the slider by decreasing the intervals between particles can further improve the quality of the simulation.
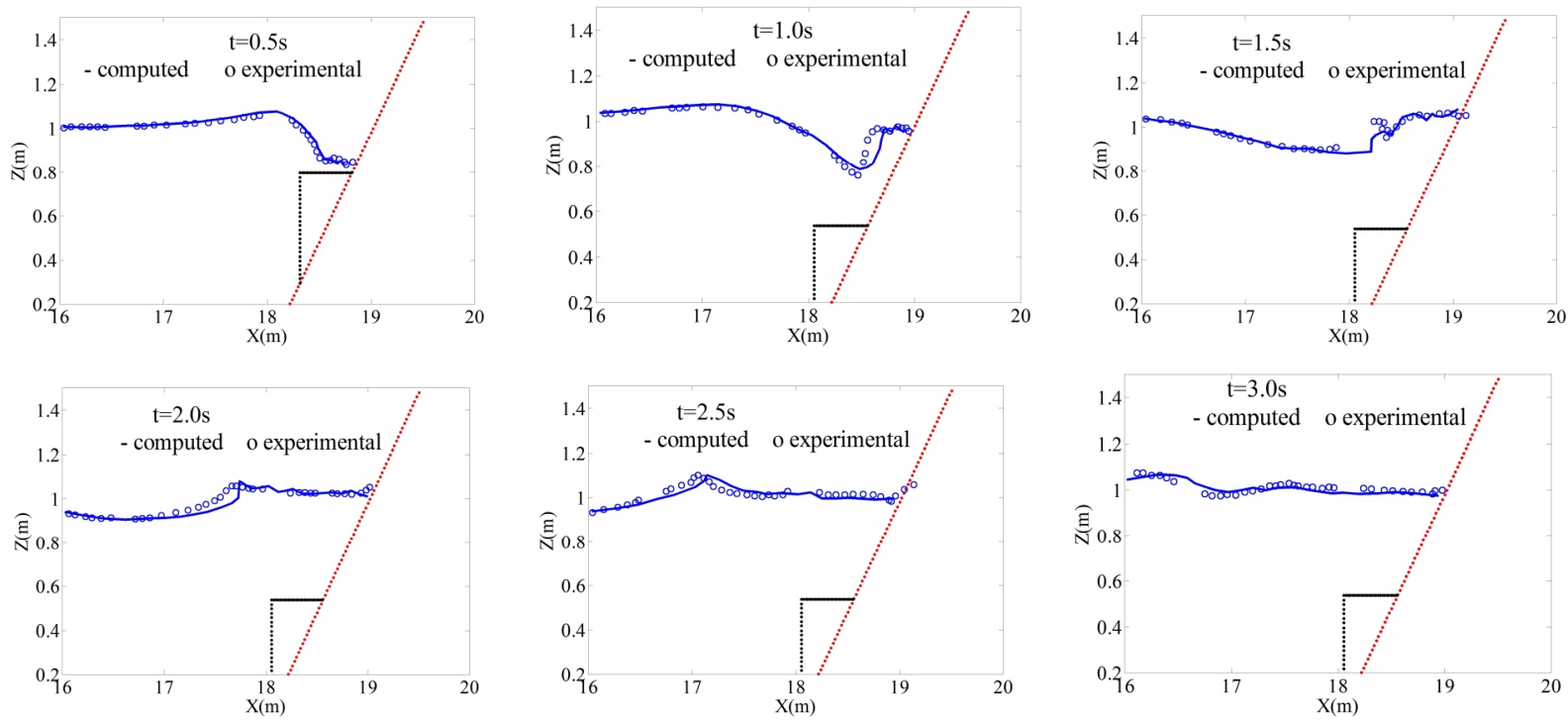

Fig. 2 Wave profiles at different moments for Submarine Landslide

Propagation of the surge wave. After being generated, the surge tends to propagate in the negative x-direction. Fig. 3 shows time-historic wave profiles acquired by the numerical simulation at the locations $x=16 \mathrm{~m}$ and $x=12 \mathrm{~m}$.
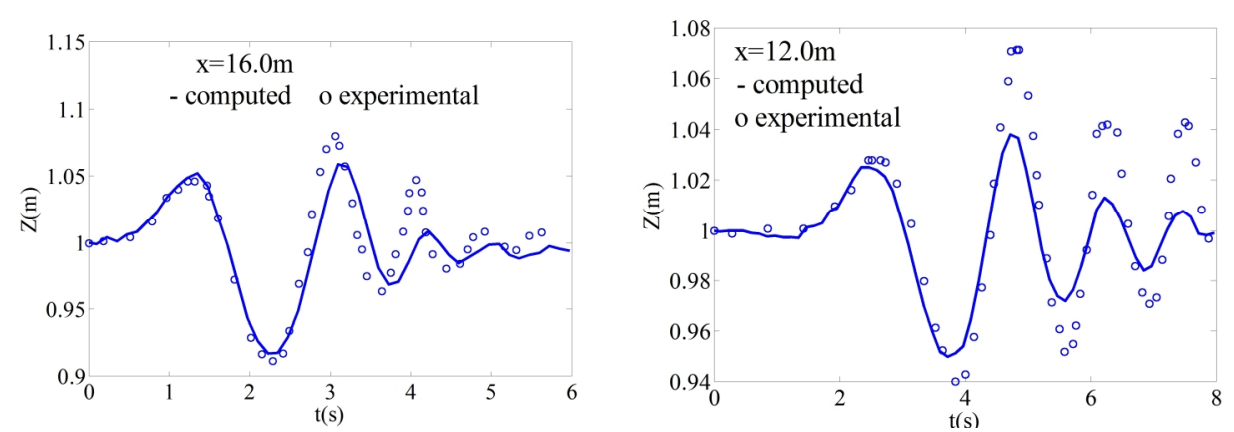

Fig. 3 Wave profiles time history for Submarine Landslide

Fig. 3 compares the experimental data and simulated results at $x=16 \mathrm{~m}$ and $x=12 \mathrm{~m}$ where the first crest and trough can be well simulated. As time goes on, the numerical amplitudes of crests and troughs attenuate faster than experimental ones, which is caused by the numerical diffusion. At the same time, the numerical wave length and wave speed agree with the experimental data. When the surge travels from $x=16 m$ to $x=12 m$, the celerity of the first crest is about $3.0 \mathrm{~m} / \mathrm{s}$, the second about $2.2 \mathrm{~m} / \mathrm{s}$. At the locations $x=16 m$ and $x=12 \mathrm{~m}$, the amplitude of the second crest is slightly greater than that of the first crest. At later times, the amplitudes of crests are reduced gradually.

Simulations of the Aerial Landslide. In the simulation of this section, the parameter settings in the SPH numerical model, including intervals between particles, density of water, motion viscosity, time step, smoothing length coefficient, etc., are the same with that for Submarine Landslide.

Generation of the surge wave. Fig. 4 is wave profiles at different time for Aerial Landslide within the region of generation of the surge by the numerical simulation. 
Fig. 4 shows the agreement between the numerical wave profiles and the experimental ones within the region of the generation of the surge. At the just moment when the slider reaches the water from the air, the number of particles, which is one of the vital factors for the error of the simulation in this part, is fewer around the slider compared with that for Submarine Landslide.
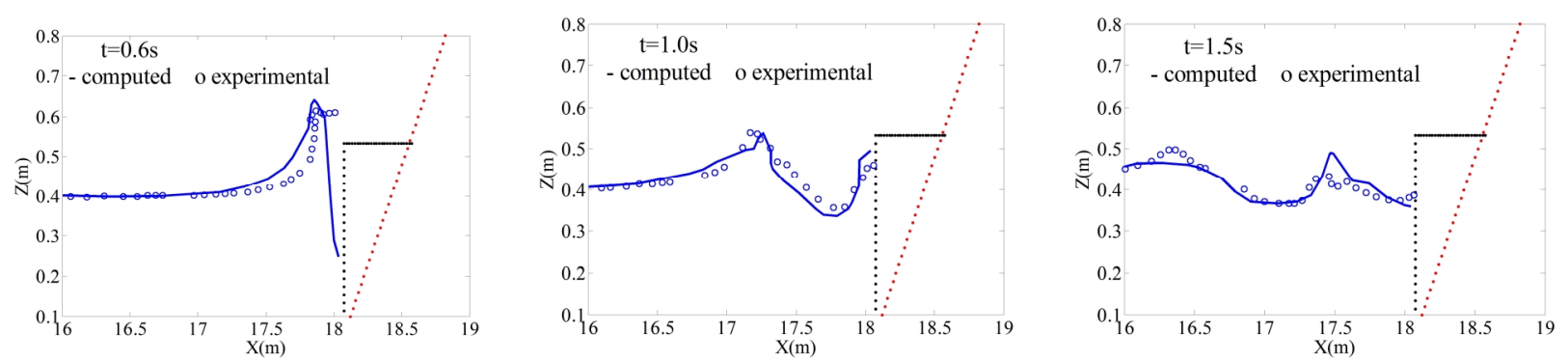

Fig. 4 Wave profiles for Aerial Landslide at different times

Propagation of the surge wave. Fig. 5 are time-historic wave profiles by the numerical simulation at $x=16 m$ and $x=12 m$.
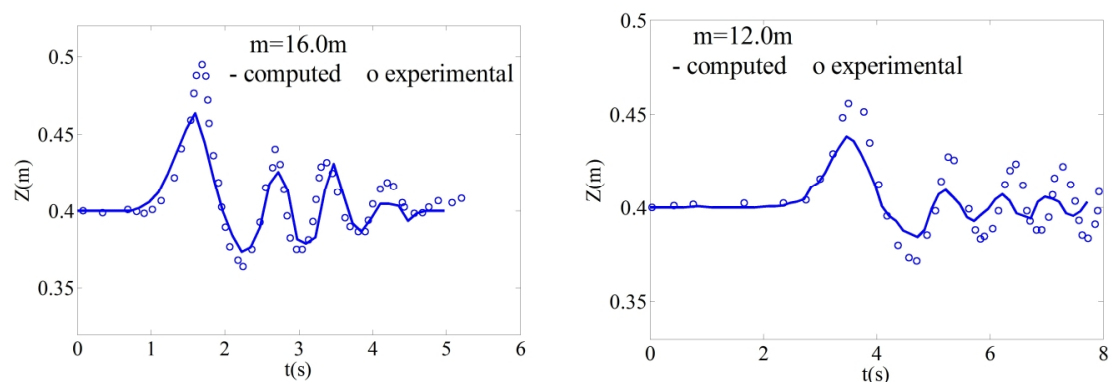

Fig. 5 Wave profiles time history for Aerial Landslide

In Fig. 5, for the simulations of wave length and wave speed, the numerical results and the experimental data are consistent. The amplitude of the first crests at $x=16 \mathrm{~m}$ and $12 \mathrm{~m}$ are both greater than the following ones. Compared with the working condition for Submarine Landslide, the calculated crests and troughs have differences with the experimental ones. Furthermore, the numerical diffusion becomes even more serious.

Simulation of different interval of particles. The quantity of particles is one of the aspects which influence the calculated diffusion of SPH. In this paper, for Aerial Landslide, additional two calculations are carried out with the intervals of particles $\Delta x=\Delta z=0.0125 \mathrm{~m}$ and $0.01 \mathrm{~m}$. Fig.6 is the comparison of wave profiles between the locations $x=16 \mathrm{~m}$ and $12 \mathrm{~m}$.
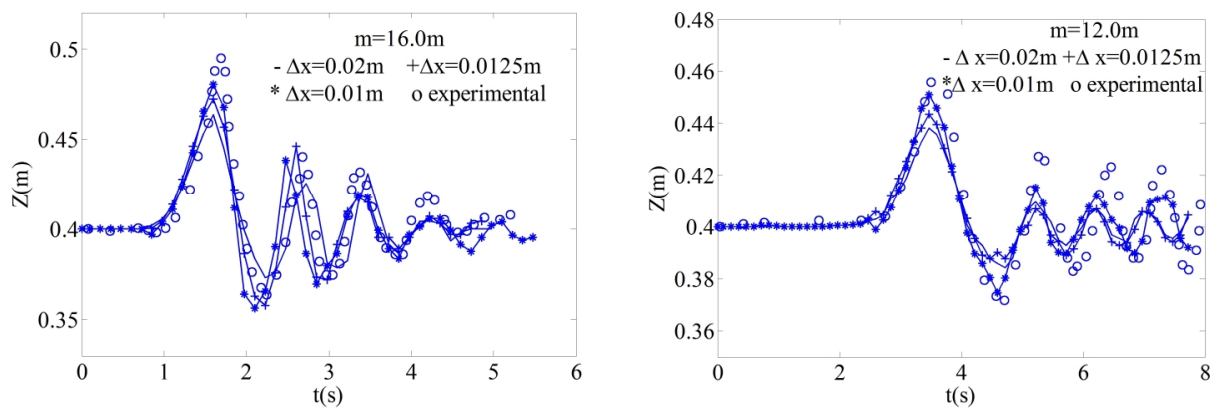

Fig.6 Time-historic wave profiles with different intervals of particles for Aerial Landslide

From Fig.6, one can see that, with the increasing of the number of particles, the calculated results of the first crest and trough at $x=16 \mathrm{~m}$ and $12 \mathrm{~m}$ are closer to the experimental ones. On one hand, it indicates that the apparent attenuation of the wave amplitudes during the propagation is directly related to the numerical diffusion. On the other hand, increasing the number of particles can improve the quality of the SPH simulation for the Landslide-generated surge. 


\section{Conclusion}

In this paper, the SPH method is used to numerically simulate the 2-D Landslide-generated surge. The results turn out that the SPH method is capable of simulating the Landslide-generated surge which has the character of strong non-linearity. When the generation of the surge is simulated, the wave profiles can be well obtained. During the simulation of the propagation of the surge, the amplitudes of the first crests and troughs, wave length and wave speed can well correspond to the experimental data. Nevertheless, due to the influence of the numerical diffusion, the simulation for the dissipation process of wave amplitudes of the surge needs to be further improved.

\section{References}

[1]Heinrich P. Nonlinear water waves generated by submarine and aerial landslides[J]. Journal of Waterway, Port, Coastal, and Ocean Engineering, 1992, 118(3): 249-266.

[2] H.M. Fritz, W.H. Hager, H.-E. Minor. Landslide generated impulse waves. [J]. Experiments in Fluids2003 (35):505-532.

[3] Chiang W. L., Divorky, D., Parnicky, P., Wier, W.. Numerical model of land-slide-generated waves. Tetra Tech Report T-3427, U.S. Department of Commerce, Pasadena, Calif.1981

[4] Gozali, S., Hunt, B..Water waves generated by close landslides. J. Hydr. Res.27(1),49-60

[5] Heinrich P..Nonlinear numerical model of landslide-generated water waves.Int. J. Engrg. Fluid Mech.,1991,4(4),403-416

[6] Shi C.Q., An Y., Yang J.X.. A SPH based numerical method of landslide induced impulse ave and its application on Huang tian landslide event. Sci Sin-Phys Mech Astron, 2015, Vol. 45, No. 4

[7] Du, X.T. , Wu, W. , Gong K. , Liu H.. Two dimensional SPH simulation of water waves generated by underwater landslide. Journal of Hydrodynamic.A. Vol,21,No.5, 2006, 579-586

[8]Lin P.Z., Liu X., Zhang J.M.. The simulation of a landslide-induced surge wave and its overtopping of a coupled ISPH model. Eng. Appl. Of Comp. Fluid Mech.2015,9:1,432-444 